\title{
Integración de prácticas culturales y control biológico para el manejo de Moniliophthora roreri Cif \& Par
}

\author{
Integration of agronomical and biological practices for the management \\ of Moniliophthora roreri Cif \& Par
}

\section{Jorge Enrique Villamil C. ${ }^{1}$; Lizeth Johana Sierra A. ${ }^{1}$; Yeimy Olarte L. ${ }^{1}$; Ana Teresa Mosquera E. ${ }^{2}$; Juan David Fajardo C. ; Elberth H. Pinzón ${ }^{4}$; John Wilson Martínez 0. ${ }^{5}$}

\footnotetext{
${ }^{1}$ Grupo Manejo Biológico de Cultivos. Universidad Pedagógica y Tecnológica de Colombia. Tunja, Colombia.

${ }^{2} \mathrm{Ph}$.D. Ciencias agrícolas. Universidad Pedagógica y Tecnológica de Colombia. Tunja, Colombia.

${ }^{3}$ Grupo Manejo Biológico de Cultivos. Universidad Pedagógica y Tecnológica de Colombia. Tunja, Colombia.

${ }^{4}$ Profesor. M.Sc. Fisiología vegetal. Universidad Pedagógica y Tecnológica de Colombia. Tunja, Colombia.

${ }^{5}$ Profesor. M.Sc. Entomología. Universidad Pedagógica y Tecnológica de Colombia. Tunja, Colombia, John.martinez@uptc.edu.co.
}

Citar: VILLAMIL, J.; SIERRA, L.; OLARTE, Y.; MOSQUERA, A.; FAJARDO, J.; PINZON, E.; MOSQUERA, E.; MARTÍNEZ, J. 2015. Integración de prácticas culturales y control biológico para el manejo de Moniliophthora roreri CIF \& PAR. Rev. Cienc. Agr. 32(2):13 -25.

\section{RESUMEN}

En Colombia las pérdidas ocasionadas por Moniliophthora roreri Cif \& Par en el cultivo de cacao, son considerables y los métodos de control tradicional no son suficientes. Para el manejo de la enfermedad el control biológico, surge como alternativa económicamente viable y ambientalmente sostenible. El objetivo del presente estudio fue evaluar el control de Moniliophthora roreri mediante parámetros de incidencia y severidad, utilizando aislamientos nativos y cepas comerciales de Trichoderma spp., en combinación con prácticas culturales (PC). Se evaluaron diez tratamientos, los cuales resultaron de combinar prácticas culturales, productos biológicos comerciales, aislamientos nativos de hongos y funguicidas de síntesis química. Para la evaluación, se empleó un diseño completamente aleatorio (DCA) con cuatro repeticiones, para un total de 40 UE cada una compuesta por una planta del clon TCS01 (Theobroma CORPOICA la Suiza 01). Se hizo inoculación dirigida al dosel, utilizando una concentración de 1,2, 1,5 y 2,9 x10 ${ }^{7}$ UFC. $\mathrm{g}^{-1}$ para aislamientos nativos y 2,0 y 5,0 x $10^{8} \mathrm{UFC} . \mathrm{g}^{-1}$ para cepas comerciales. Se evaluó incidencia y severidad externa en los frutos durante 25 semanas. Los resultados indicaron que los frutos inoculados con el tratamiento T7 (P.C + Safer soil @W.P) y T4 (P.C + mezcla Trichoderma H20, H3, H5), presentaron los menores porcentajes de incidencia y severidad externa y los mayores rendimientos de cosecha sin diferencias significativas respecto al control químico y regional, representando una alternativa promisoria para la producción de cacao en la subregión occidental de Boyacá.

Palabras clave: Cacao, Moniliasis, Trichoderma, biocontrol, aislamientos nativos. 


\begin{abstract}
In Colombia, yield loses in cocoa, due to the Frosty Pod Rot Moniliophthora roreri Cif \& Pa, are significant and the traditional methods of control are not enough. For the disease management, the biological control, therefore, emerges as an economically viable and environmentally sustainable alternative. The objective of this study was to evaluate Moniliophthora roreri control's by analyzing incidence and severity parameters, using native and commercial strains of Trichoderma spp., combined with agronomic practices (AP). Ten treatments were evaluated. They included: agronomic practices, biological products, native fungi strains, and chemical fungicide combinations. A completely randomized design with four replicates and 40 experimental units was used. Each unit consisted of a plant of the TCS01 (Theobroma Corpoica La Suiza 01) variety. Foliage inoculations were done using concentrations of 1.2, 1.5, and 2,9 x107 UFC.g-1for native fungal strains and 2,0 and 5.0 x108 UFC.g-1 concentrations for commercial strains . External incidence and severity on fruits were evaluated during 25 weeks. Results showed that fruits inoculated with treatment T7 (AP + Safer soil W.P®) and treatment T4 (AP +mixture of Trichoderma $\mathrm{H} \mathrm{0,} \mathrm{H3,} \mathrm{H5)} \mathrm{presented} \mathrm{the} \mathrm{lowest} \mathrm{external} \mathrm{incidence} \mathrm{and}$ severity percentages and the highest yield, without significant differences between the chemical and regional control. According to the results, these treatments represent a promising alternative for cocoa production in the western subregion of Boyacá.
\end{abstract}

Keywords: Cocoa, Frosty pod rot, Trichoderma, biocontrol, native strains.

\section{INTRODUCCIÓN}

El cultivo de cacao Theobroma cacao L. se proyecta como una de las mejores opciones productivas para diferentes zonas agroecológicas colombianas, debido a la creciente demanda del mercado tanto interno como externo, además, por el incremento del precio del grano seco en el mercado internacional, el cual cerró en 3,219 USD.t ${ }^{-1}$ para julio de 2014, el nivel más alto que ha tenido en los últimos tres años según la bolsa de New York (FEDECACA0, 2015).

De acuerdo con la Corporación Colombiana de Investigación Agropecuaria (CORPOICA) y la Federación Nacional de Cacaoteros (FEDECACA0), en estudios de zonificación de suelos y clima, para el desarrollo del cultivo en el año 2005, se concluyó que en Colombia existen dos millones de hectáreas aptas para plantaciones de cacao (PROEXPORT, 2012). Sin embargo, la producción y calidad del grano, se ve afectada por problemas fitosanitarios, entre ellos las enfermedades ocasionadas por hongos fitopatógenos (Hebbar, 2007).

La moniliasis es una enfermedad causada por el hongo Moniliophthora roreri Cif \& Par, impacta el $40 \%$ de la producción anual de cacao en Colombia dependiendo de las condiciones ambientales, manejo del cultivo y de los genotipos sembrados (Jaimes y Aranzazu, 2010; Jaimes et al., 2011). Esto puede equivaler en términos de pérdidas de grano comercial a 28.000 TM sobre una producción de 42.000 (Rodríguez et al., 2005).

Especies antagonistas del género Trichoderma spp. tienen la capacidad de actuar contra una amplia variedad de patógenos del suelo como (Fusarium spp., Rhizoctonia spp., Pythium spp., Phytophthora spp. y Sclerotinia sp.) y del follaje (Botrytis spp. y Peronospora sp.) (Reyes et al., 2008; Rodríguez et al., 2010; Guerrero et al., 2012; Martínez et al., 2013). No obstante, la evaluación del control biológico de la moniliasis, aún se encuentra en una fase experimental (Krauss et 
al., 2010). Las especies de los géneros Trichoderma y Clonostachys, han sido ampliamente calificadas como agentes eficaces en el biocontrol de la enfermedad, tanto en condiciones In vitro como In situ, en diferentes áreas geográficas de Perú, Colombia, Costa rica y Ecuador (Hebbar, 2007; Bailey et al., 2008; Osorio, 2010; Krauss et al., 2010; Villamil, 2011; Villamil et al., 2012; Suárez y Rangel, 2013; Hernández et al., 2014; Villamil et al., 2015). Teniendo en cuenta lo anterior, el objetivo de esta investigación fue la evaluación del control de $M$. roreri tomando como parámetros incidencia y severidad de la enfermedad, al utilizar aislamientos nativos y cepas comerciales de Trichoderma spp., en combinación con prácticas culturales, debido a que los antagonistas microbianos, ejercen su control a largo plazo, integrado con diferentes prácticas agronómicas. También se contrastó la aplicación de Óxido de Cobre y el control regional, con la finalidad de incluir el componente biológico dentro del manejo integrado de $M$. roreri en el cultivo de cacao para la subregión occidente del departamento de Boyacá.

\section{MATERIALES Y MÉTODOS}

Ubicación del estudio. La investigación se realizó en la finca Los Sitios, del municipio de Coper, Boyacá, coordenadas 0529’30.2”'LN,

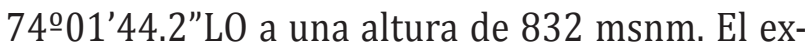
perimento se desarrolló a una temperatura promedio de $24,7^{\circ} \mathrm{C}$, precipitación pluvial acumulada de 1,254 mm y humedad relativa de $70 \%$. El estudio se llevó a cabo durante el ciclo de producción del segundo semestre de 2014.

Definición de tratamientos. Los tratamientos evaluados se constituyeron por: tres aislamientos nativos de Trichoderma spp. H3, H5 y H20; tres cepas comerciales identificadas como: Trombo ${ }^{\circledR}$ W.P (i.a. T. harzianum + T. lignorum $+T$. viride + Saccharomyces cerevisiae), ${ }^{\circledR}$ Protector(i.a T. harzianum) y Safer Soil ${ }^{\circledR}$ W.P (i.a T. asperellum, T. atroviride, T. harzianum y Paecilomyces lilacinus) y como control químico Oxido de cobre, en integración con prácticas culturales (Tabla 1).

Tabla 1. Productos evaluados para el control de moniliasis en frutos de cacao en condiciones de campo. Municipio de Coper-Boyacá, Colombia.

\begin{tabular}{|c|c|c|}
\hline Tratamiento/Producto & Formulación & Concentración \\
\hline T1. Trichoderma sp. H20 & formulación sólida & $1,5 \times 10^{7}$ UFC.g-1 \\
\hline T2. Trichoderma sp. H3 & formulación sólida & $1,2 \times 10^{7}$ UFC.g $^{-1}$ \\
\hline T3. Trichoderma sp. H5 & formulación sólida & $2.9 \times 10^{7}$ UFC. $^{-1}$ \\
\hline T4. Mezcla aislamientos nativos & formulación sólida & 1,8x107 UFC.g-1 \\
\hline T5. Trombo ${ }^{\circledR} \mathrm{WP}$ & polvo mojable (WP) & $2,0 \times 10^{8}$ UFC.g $^{-1}$ \\
\hline T6. Protector ${ }^{\circledR} \mathrm{WP}$ & polvo mojable (WP) & $2,0 \times 10^{8}$ UFC. $g^{-1}$ \\
\hline T7. Safer Soil ${ }^{\circledR}$ WP & polvo mojable (WP) & $5,0 \times 10^{8}$ UFC. $^{-1}$ \\
\hline T8. Mezcla cepas comerciales & polvo mojable (WP) & $3,0 \times 10^{8}$ UFC.g- ${ }^{-1}$ \\
\hline T9. Oxicloruro cobre & polvo mojable (WP) & 58,8\% del i.a. \\
\hline T10. Control regional & NA & NA \\
\hline
\end{tabular}


Las prácticas culturales (P.C), consistieron en una poda de mantenimiento al dosel del cacao al inicio del estudio y otra a los seis meses siguientes; una poda al dosel del sombrío permanente, manejo de arvenses como Brachiaria decumbens, Paspalum millegrana Schrad y Sida acuta Burm. f. con periocidad trimestral, fertilización edáfica con base al análisis de suelos para la finca y remoción de frutos afectados por M. roreri a intervalos quincenales para los tratamientos inoculados con antagonistas microbianos y el control químico. En el control regional la remoción se hizo semanalmente (RESE).

Diseño experimental. Se utilizó un diseño completamente aleatorio (DCA) con 10 tratamientos y cuatro repeticiones, para un total de 40 unidades experimentales, cada una compuesta por un árbol del Clon "Theobroma CORPOICA la Suiza 01" (TCS $01)$ de 2,5 años de edad. El material vegetal se seleccionó por presentar susceptibilidad a $M$. roreri dentro de un cultivo de 2,5 ha con distancia de siembra de $3 \mathrm{~m} \times 3 \mathrm{~m}$, establecido en triangulo y una densidad de 1,100 árboles.ha ${ }^{-1}$ en arreglo forestal con Teca (Tectona grandis) y Cedro nativo (Cedrela odorata) que aportan el 30\% de sombra para el sistema (Jaimes y Aranzazu, 2010).

Obtención de inoculantes microbianos nativos. Los aislamientos nativos de Trichoderma spp. H3, $\mathrm{H} 5 \mathrm{y} \mathrm{H}_{2} \mathrm{O}$ se obtuvieron previamente de suelo rizosférico y de frutos de cacao de árboles procedentes de semilla, de diversos cruzamientos interclonales del municipio de Coper (Boyacá) y fueron seleccionados por su potencial para reducir el crecimiento y esporulación de M. roreri en condiciones In vitro e In situ (Villamil, 2011; Villamil et al., 2012, 2015). Se incubaron a $25^{\circ} \mathrm{C}$ por 8 días, en cajas Petri que contenían medio de cultivo Agar Papa Dextrosa ( $\left.{ }^{\circledR P D A}, \mathrm{ASIMEL}\right)$, suplementado con un antibiótico (i.a. ${ }^{\circledR}$ cloranfenicol MK) para evitar la contaminación bacteriana (Lucero et al., 2004).

Producción masiva del inóculo. Se emplearon bolsas de polipropileno a las cuales se adicionaron 100 g de arroz blanco y $90 \mathrm{~mL}$ de agua destilada estéril (A.D.E.). El medio de cultivo se esterilizó en autoclave a $121^{\circ} \mathrm{C}, 15$ PSI por 25 minutos. Para la siembra se tomó un trozo de $0,5 \mathrm{~cm}$ del medio PDA colonizado por el antagonista microbiano y se introdujo en las bolsas que contenían arroz estéril. Las bolsas inoculadas fueron incubadas a $25^{\circ} \mathrm{C} \mathrm{du}$ rante 25 días según la metodología empleada por CENICAFE (1994).

Obtención de la concentración de UFC. Se preparó una suspensión de cada uno de los aislamientos nativos de Trichoderma spp. H3, H5 y H20 en A.D.E en un patrón de $9 \mathrm{~mL}$, utilizando $1 \mathrm{~g}$ de arroz precocido colonizado por el biocontrolador y 50 $\mu \mathrm{L}$ de ${ }^{\circledR}$ Tween 80 (ASIMEL), para la dispersión de las conidios (Vélez et al., 1997). De esta suspensión se hicieron diluciones seriadas hasta $1 \times 10^{-3} \mathrm{y}$ se tomaron $50 \mu \mathrm{L}$ para hacer los conteos en cámara neubauer con ayuda de un microscopio Nikon ${ }^{\circledR}$ (Vélez et al., 1997).

Inoculación de antagonistas microbianos y el fungicida. Para evitar la contaminación entre antagonistas microbianos, el oxicloruro de cobre y el control regional, se distanciaron las unidades experimentales dejando 2 plantas y un surco de clones de cacao entre arboles de TCS01. Las aplicaciones fueron hechas al inicio de floración, cuajado y crecimiento de pepinos, con una dosis de 200 m. \pm ,2/árbol. La inoculación de biocontroladores se realizó en horas de la tarde dirigida al dosel, cojines florales y frutos en diferentes estados de desarrollo, usando el método de inundación masiva (Jaimes y Aranzazu, 2010). El fungicida fue aplicado mediante aspersión localizada a frutos en diferentes estados de desarrollo.

Variables de respuesta. Se llevó un registro semanal de incidencia y severidad externa de la enfermedad, por un periodo de 25 semanas. La incidencia de la enfermedad en cada semana se calculó en porcentaje mediante la fórmula de Argüello (2000), contando el número de frutos con 
el síntoma de la enfermedad. La severidad externa se evaluó, primero con base en el porcentaje de tejido afectado en fruto, utilizando la escala dada en grados y porcentaje de afectación (Grado 1= 0\% fruto sano, Grado 2=1-25\% protuberancia, Grado $3=26-50 \%$ inicio de mancha, Grado $4=51-75 \%$ mancha, Grado 5= 76-100\% esporulación); luego mediante la sumatoria de los grados de afectación en porcentaje sobre el número total de datos por el mayor dato del porcentaje de severidad (Cárdenas y Giraldo, 1986). Para comparar el efecto de los tratamientos sobre la incidencia y severidad externa se calculó el área bajo la curva del progreso de la enfermedad (ABCPE) mediante la aplicación de la fórmula establecida por Shaner y Finney (1977), con base en la metodología planteada por Pérez y Forbes (2008). Los datos analizados correspondieron a: porcentaje de incidencia y severidad externa, teniendo en cuenta el número del día, en el cual se hizo la evaluación después de la aplicación de los tratamientos. Finalmente, se determinó la producción de cacao en grano seco por hectárea, mediante cosecha quincenal para las 25 semanas de evaluación.
Análisis estadístico. Los datos obtenidos fueron sometidos al análisis de normalidad y homogeneidad de varianza para cada variable, mediante la prueba de Kolmogorov-Smirnov y Levene (Gómez y Gómez, 1984). A las variables que cumplieron los supuestos se les realizó Análisis de Varianza (ANDEVA) y se les aplicó las pruebas de comparación múltiple de Duncan, utilizando el paquete estadístico SAS v. 9,2e ${ }^{\odot}$ (Cary, N.C). Las variables que no cumplieron los supuestos de normalidad y homocedasticidad y que no pudieron ser transformadas, se analizaron mediante la prueba no paramétrica de Kruskal Wallis, usando el programa estadístico InfoStat $\mathrm{L}^{\odot}$ (IS-UNC).

\section{RESULTADOS Y DISCUSIÓN}

Incidencia de moniliasis en frutos de cacao. El ANDEVA indicó diferencias significativas entre tratamientos $(\mathrm{P}=0,01)$ en los meses dos y tres. La prueba de Kruskal-Wallis $(\mathrm{P}=0,01)$ mostró diferencias significativas entre tratamientos en los meses cuatro y cinco. En el primer mes no se presentaron diferencias entre tratamientos.

Tabla 2. Efecto de los tratamientos evaluados sobre la incidencia de moniliasis en frutos de cacao en condiciones de campo. Municipio de Coper-Boyacá, Colombia.

\begin{tabular}{llllll}
\hline \multicolumn{1}{c}{ Tratamiento } & \multicolumn{5}{c}{ Incidencia mensual (\%) } \\
\hline \multicolumn{1}{c}{$\mathbf{1}$} & \multicolumn{1}{c}{$\mathbf{2}$} & \multicolumn{1}{c}{$\mathbf{3}$} & \multicolumn{1}{c}{$\mathbf{4}^{*}$} & \multicolumn{1}{c}{$\mathbf{5}^{*}$} \\
\hline 1. P.C + Trichoderma spp. H20 & $13,9 \pm 3,7 \mathrm{ab}$ & $9,7 \pm 3,3 \mathrm{c}$ & $7,1 \pm 1,6 \mathrm{c}$ & $5,5 \pm 0,6 \mathrm{abc}$ & $0,0 \mathrm{a}$ \\
2. P.C + Trichoderma spp. H3 & $15,4 \pm 6,1 \mathrm{ab}$ & $24,3 \pm 2,2 \mathrm{a}$ & $13,8 \pm 1,1 \mathrm{~b}$ & $40,6 \pm 8,9 \mathrm{c}$ & $0,0 \mathrm{a}$ \\
3. P.C + Trichoderma spp.H5 & $5,5 \pm 1,3 \mathrm{~b}$ & $17,0 \pm 0,2 \mathrm{~b}$ & $6,9 \pm 2,0 \mathrm{c}$ & $4,6 \pm 0,4 \mathrm{ab}$ & $0,0 \mathrm{a}$ \\
4. P.C + Trichoderma H20, H3, H5 & $11,2 \pm 4,7 \mathrm{ab}$ & $9,1 \pm 1,5 \mathrm{c}$ & $7,7 \pm 1,2 \mathrm{bc}$ & $0,0 \mathrm{a}$ & $0,0 \mathrm{a}$ \\
5. P.C + Trombo ${ }^{\circledR}$ W.P & $17,1 \pm 7,4 \mathrm{ab}$ & $12,4 \pm 3,1 \mathrm{bc}$ & $9,0 \pm 2,8 \mathrm{bc}$ & $5,9 \pm 0,9 \mathrm{abc}$ & $0,0 \mathrm{a}$ \\
6. P.C + Protector ${ }^{\circledR}$ W.P & $11,1 \pm 0,4 \mathrm{ab}$ & $19,5 \pm 2,3 \mathrm{ab}$ & $8,9 \pm 0,7 \mathrm{bc}$ & $0,0 \mathrm{a}$ & $9,0 \pm 2,1 \mathrm{~b}$ \\
7. P.C + Safer soil ${ }^{\circledR}$ W.P & $18,6 \pm 2,4 \mathrm{ab}$ & $0,0 \mathrm{~d}$ & $4,8 \pm 0,5 \mathrm{c}$ & $0,0 \mathrm{a}$ & $0,0 \mathrm{a}$ \\
8. P.C + mezcla cepas comerciales & $27,9 \pm 5,9 \mathrm{a}$ & $13,9 \pm 1,3 \mathrm{bc}$ & $26,4 \pm 4,2 \mathrm{a}$ & $0,0 \mathrm{a}$ & $0,0 \mathrm{a}$ \\
9. P.C + Control químico & $10,8 \pm 3,0 \mathrm{ab}$ & $12,8 \pm 3,8 \mathrm{bc}$ & $4,5 \pm 0,7 \mathrm{c}$ & $18,6 \pm 2,3 \mathrm{bc}$ & $6,0 \pm 0,8 \mathrm{~b}$ \\
10. Control regional & $22,0 \pm 5,8 \mathrm{ab}$ & $9,4 \pm 0,3 \mathrm{c}$ & $5,1 \pm 0,8 \mathrm{c}$ & $20,2 \pm 4,8 \mathrm{bc}$ & $7,3 \pm 2,0 \mathrm{~b}$ \\
\hline
\end{tabular}

Promedios seguidos por letras distintas, indican diferencias significativas entre tratamientos según la prueba (Duncan; $\mathrm{P}<, 01$ ), *rangos seguidos de letras distintas indican diferencias significativas entre tratamientos para la incidencia en los meses cuatro y cinco (Kruskal Wallis; $\mathrm{P}<0,01$ ). Valores a la derecha de la media indican el error estándar. 
En la octava semana (mes dos), el T7 (P.C + Safer soil ${ }^{\circledR}$ W.P) mostró diferencias significativas en comparación con los demás tratamientos y el testigo regional (T10), lo cual se interpreta como control del patógeno que se refleja en el porcentaje de incidencia de moniliasis. En este mismo mes, el T4 (P.C + mezcla aislamientos autóctonos), también presentó diferencias respecto a los demás tratamientos, pero no frente al T9 (P.C + control químico) y T10. A partir de la semana dieciséis (mes 4), los tratamientos compuestos por aislamientos autóctonos y cepas comerciales de Trichoderma spp., mostraron diferencias significativas frente al T9 y T10. Los tratamientos que tuvieron una mejor respuesta en la evaluación al quinto mes fueron T1, T2, T3, T4, T5, T7 y T8. Los demás tratamientos tuvieron un efecto muy similar (Tabla 2). De acuerdo con Phillips et al. (2007), la espora de M. roreri necesita de la presencia de agua para germinar, por lo que la enfermedad se incrementa en los meses de lluvia. En general, el hongo requiere una precipitación anual de 780 a 5,500 mm, temperatura promedio anual de 18,6 a $28{ }^{\circ} \mathrm{C}$ y $85 \%$ de humedad relativa (Phillips y Wilkinson, 2007; Phillips et al., 2007), condiciones que se corresponden con las óptimas de crecimiento del patógeno.

Área bajo la curva del progreso de la enfermedad (ABCPE). El ANDEVA mostró diferencias significativas entre tratamientos. Los valores del ABCPE para cada tratamiento fueron: T1: 986, T2: 2834, T3: 943, T4: 675 T5: 1157, T6: 1156, T7: 424, T8: 1629, T9: 1331 y T10: 1490. El mayor valor expresado por el T2, se debió a que las unidades experimentales de este, coincidieron con el área más sombreada del lote lo que ocasionó aumento en la humedad relativa por encima del $75 \%$, como resultado de las altas precipitaciones y temperaturas mayores a $23^{\circ} \mathrm{C}$ en promedio para la zona durante los seis meses de evaluación, condiciones óptimas para el desarrollo de M. roreri (Phillips et al., 2007). En la Figura 1, los valores más bajos corresponden a los tratamientos con mayor efecto de biocontrol sobre la incidencia de moniliasis. No hubo diferencias significativas entre $\mathrm{T} 7 \mathrm{y} \mathrm{T}$, pero si frente a los demás tratamientos y el control regional (T10). El T4, mostró diferencias en relación con los tratamientos T2, T8, T9 y T10.

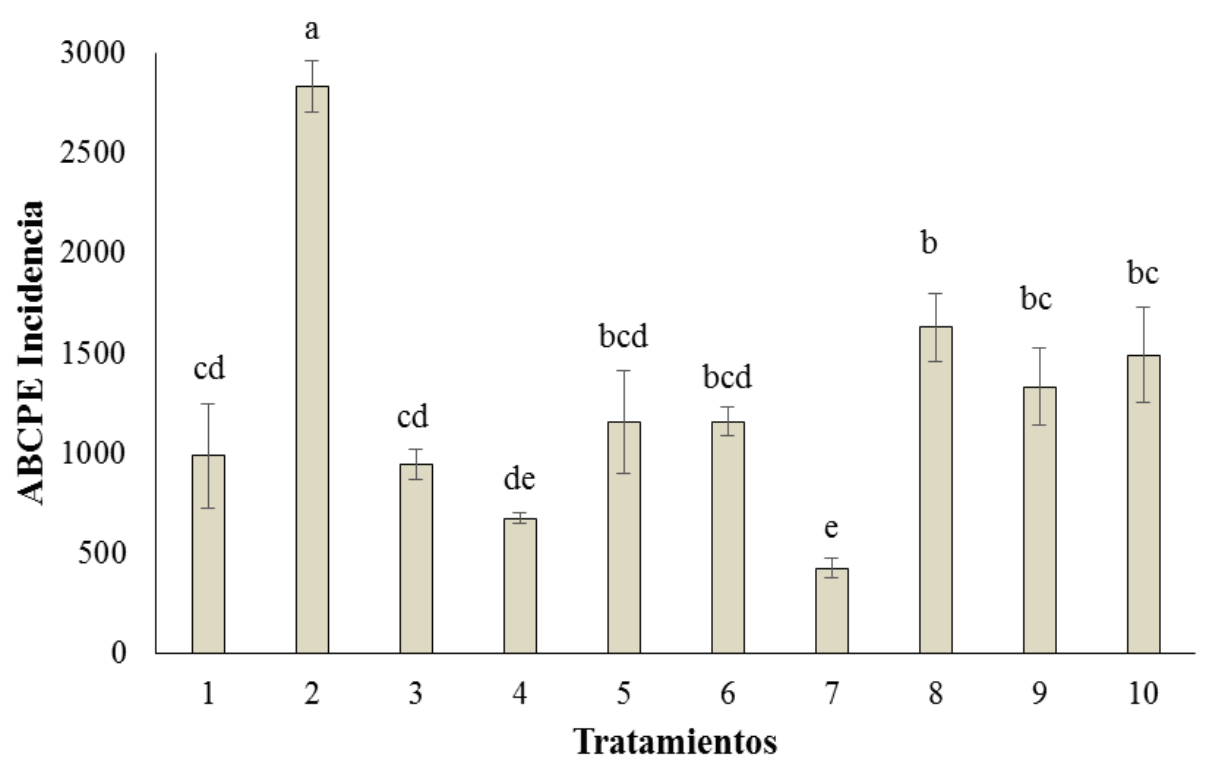

Figura 1. Área bajo la curva del progreso de la enfermedad (ABCPE) con base a incidencia de $M$. roreri en respuesta a los tratamientos evaluados. Letras distintas indican diferencias significativas según Duncan $(\mathrm{P} \leq 0,01)$. Los intervalos sobre cada barra representan el error estándar. 


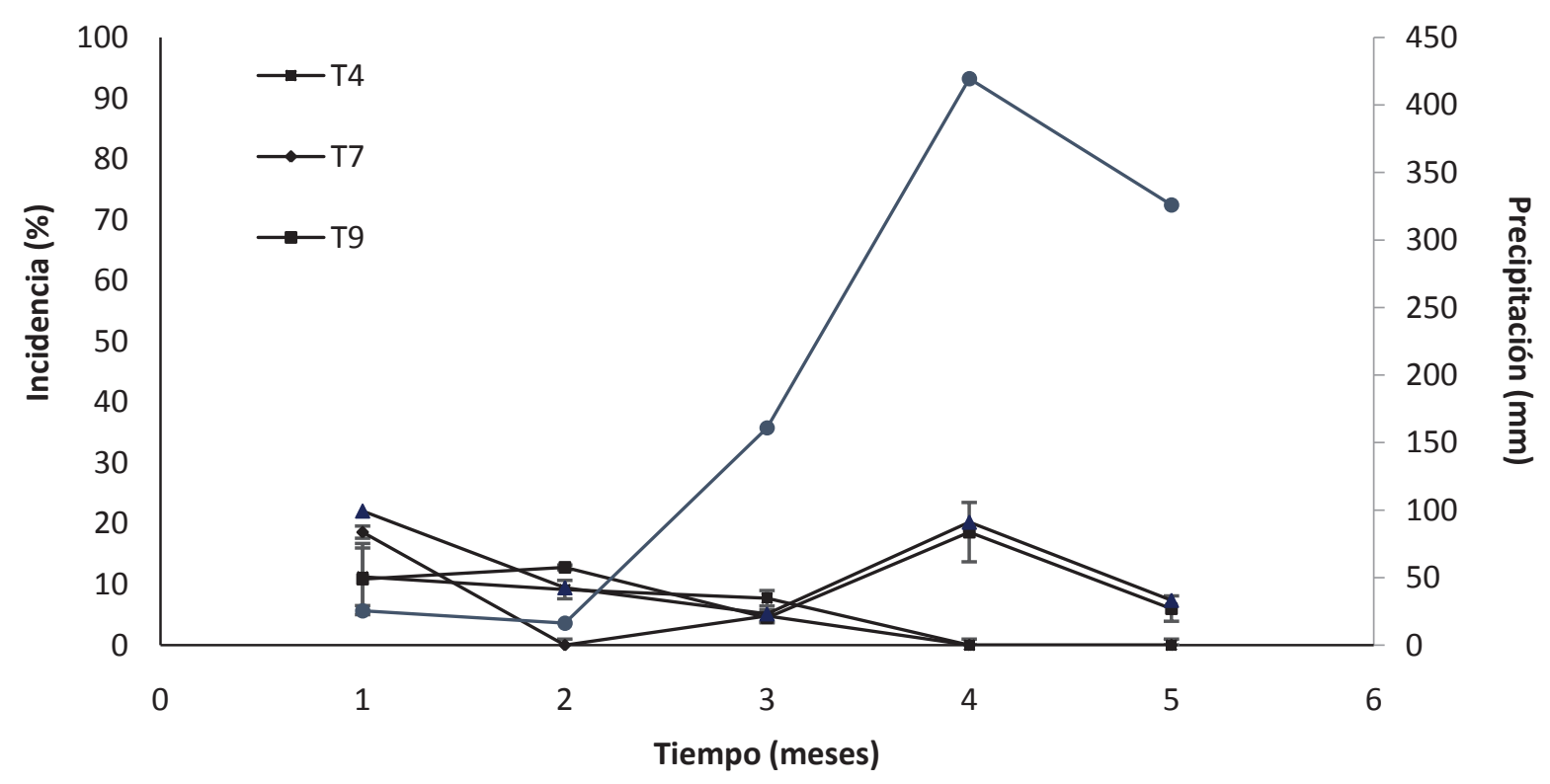

Figura 2. Relación entre la incidencia de moniliasis sobre frutos de cacao y la precipitación en el tiempo, en presencia de los tratamientos T4, T7, control químico y testigo regional en condiciones de campo. Las barras sobre la curva representan el error estándar.

Al analizar la incidencia de la enfermedad a través del tiempo por los valores expresados, se confirmó, que el mayor control durante las veinticinco semanas del experimento se obtuvo con el T7 seguido del T4, que superan a T9 y T10 (Figura 2). Entre tanto, los tratamientos con el menor efecto de control fueron T2 y T8, seguidos de T10, T9, T5, T6, T1 y T3 (Figura 1). Estos resultados están de acuerdo con Martínez et al. (2013), quienes afirman que el control efectuado por Trichoderma spp depende más de las cepas, que de la especie, pues éstas pueden presentar diferencias en sus modos de acción, aun perteneciendo a una misma especie. Esto refuerza la necesidad de efectuar una correcta selección de los aislamientos respecto a sus ambientes, para obtener resultados consistentes en condiciones de campo (Martínez et al., 2008).

Estos resultados corroboran lo mencionado por Solis y Suárez (2005), al indicar que se pueden utilizar aislamientos autóctonos de Trichoderma spp. en combinación con prácticas culturales para mantener bajos los niveles de la enfermedad inducida por $M$. roreri. Al respecto Bateman et al. (2005) afirman que el efecto de las aplicaciones de agentes biológicos, no es comparable con el de los fungicidas, debido a su diferente modo de acción y selectividad. En el corto plazo, los bioantagonistas pueden resultar costosos y tomar más tiempo para ejercer su control, pero esto es compensado por su establecimiento en el agrosistema con un prolongado periodo de control y mayor selectividad sobre la microbiota benéfica (Krauss et al., 2010).

Severidad externa. Para ésta variable el ANDEVA no mostró diferencias significativas entre tratamientos en los meses uno y tres; contrario ocurrió en el mes dos $(\mathrm{P}=0,01)$. La prueba Kruskal-Wallis $(\mathrm{P}=0,01)$ presento diferencias significativas entre rangos de los tratamientos en los meses cuatro y cinco (Tabla 3.). 
Tabla 3. Efecto de los tratamientos evaluados sobre la severidad externa de moniliasis en frutos de cacao en condiciones de campo. Municipio de Coper-Boyacá, Colombia.

\begin{tabular}{llcccl}
\hline \multicolumn{1}{c}{ Tratamiento } & \multicolumn{5}{c}{ Severidad Externa mensual (\%) } \\
\hline \multicolumn{1}{c}{1} & \multicolumn{1}{c}{2} & \multicolumn{1}{c}{3} & $4^{*}$ & $5^{*}$ \\
\hline 1. P.C + Trichoderma spp. H20 & $48,4 \pm 1,0 \mathrm{ab}$ & $60,9 \pm 8,8 \mathrm{bc}$ & $48,4 \pm 0,6 \mathrm{ab}$ & $50,0 \pm 0,0 \mathrm{~b}$ & $0,0 \mathrm{a}$ \\
2. P.C + Trichoderma spp. H3 & $63,3 \pm 13,9 \mathrm{ab}$ & $49,1 \pm 3,0 \mathrm{bc}$ & $69,0 \pm 7,7 \mathrm{a}$ & $62,9 \pm 8,2 \mathrm{~b}$ & $0,0 \mathrm{a}$ \\
3. P.C + Trichoderma spp.H5 & $57,3 \pm 12,8 \mathrm{ab}$ & $84,1 \pm 6,4 \mathrm{a}$ & $70,8 \pm 12,5 \mathrm{a}$ & $66,6 \pm 11,7 \mathrm{~b}$ & $0,0 \mathrm{a}$ \\
4. P.C + Trichoderma H20, H3, H5 & $73,3 \pm 14,0 \mathrm{a}$ & $48,8 \pm 9,8 \mathrm{bc}$ & $59,8 \pm 9,7 \mathrm{ab}$ & $0,0 \mathrm{a}$ & $0,0 \mathrm{a}$ \\
5. P.C + Trombo ${ }^{\circledR}$ W.P & $43,2 \pm 3,9 \mathrm{~b}$ & $51,4 \pm 5,9 \mathrm{bc}$ & $65,4 \pm 10,9 \mathrm{ab}$ & $63,8 \pm 11,7 \mathrm{~b}$ & $0,0 \mathrm{a}$ \\
6. P.C + Protector ${ }^{\circledR}$ W.P & $66,8 \pm 6,8 \mathrm{ab}$ & $43,3 \pm 8,0 \mathrm{bc}$ & $37,0 \pm 5,5 \mathrm{~b}$ & $0,0 \mathrm{a}$ & $37,5 \pm 5,1 \mathrm{~b}$ \\
7. P.C + Safer soil ${ }^{\circledR}$ W.P & $66,9 \pm 6,9 \mathrm{ab}$ & $0,0 \mathrm{~d}$ & $43,0 \pm 2,8 \mathrm{ab}$ & $0,0 \mathrm{a}$ & $0,0 \mathrm{a}$ \\
8. P.C + mezcla cepas comerciales & $72,5 \pm 7,5 \mathrm{ab}$ & $62,5 \pm 5,1 \mathrm{~b}$ & $48,8 \pm 10,9 \mathrm{ab}$ & $0,0 \mathrm{a}$ & $0,0 \mathrm{a}$ \\
9. P.C + Control químico & $59,3 \pm 3,8 \mathrm{ab}$ & $54,5 \pm 1,8 \mathrm{bc}$ & $50,0 \pm 0,0 \mathrm{ab}$ & $47,0 \pm 4,5 \mathrm{~b}$ & $41,6 \pm 5,8 \mathrm{~b}$ \\
10. Control regional & $60,6 \pm 5,5 \mathrm{ab}$ & $42,0 \pm 3,2 \mathrm{c}$ & $56,2 \pm 12,7 \mathrm{ab}$ & $32,3 \pm 8,4 \mathrm{ab}$ & $25,0 \pm 0,0 \mathrm{~b}$ \\
\hline
\end{tabular}

Promedios seguidos por letras distintas, indican diferencias significativas entre tratamientos según la prueba (Duncan; $\mathrm{P}<0,01$ ), * rangos seguidos de letras distintas indican diferencias significativas entre tratamientos para la severidad externa en los meses cuatro y cinco (Kruskal Wallis; $\mathrm{P}<0,01$ ). Valores a la derecha de la media indican el error estándar.

En la octava semana (mes dos) el tratamiento T7 (P.C + Safer soil ${ }^{\circledR}$ W.P), fue diferente significativamente frente a los demás tratamientos y el control regional con ausencia de tejido externo afectado en los frutos; igual ocurrió con T3 (P.C + Trichoderma spp. H5), que presentó diferencias respecto a los demás tratamientos. A partir de la semana dieciséis (mes cuatro) los tratamientos T4, T6, T7 y T8 inhibieron la afección del patógeno en tejido externo de frutos con diferencias significativas, frente a los otros tratamientos. Los tratamientos que tuvieron una mejor respuesta en la evaluación al quinto mes fueron T1, T2, T3, T4, T5, T7 y T8. Los demás tratamientos tuvieron un efecto muy similar (Tabla 3).

Área bajo la curva del progreso de la enfermedad (ABCPE). Según el ANDEVA, existieron diferencias significativas entre tratamientos $(\mathrm{P}=$ $0,01)$. Los valores del ABCPE para cada tratamiento fueron: T1:5507, T2:6515, T3:7510, T4: 4360, T5:6070, T6:3975, T7:2296, T8:4428, T9:6063 у T10:5204. Los valores más bajos corresponden a los tratamientos con mayor efecto de biocontrol sobre la severidad externa de moniliasis. La mayor severidad de los tratamientos T2:6515, T3:7510 y T5:6070, se debió probablemente a que en éstos, se hizo la recolección de mazorcas enfermas con periocidad quincenal respecto al testigo regional con frecuencia semanal, sumado a una lenta adaptación de los antagonistas microbianos al microclima de la zona. En las interacciones antagónicas de Trichoderma spp pueden estar involucrados diferentes mecanismos de acción, los cuales dependen del aislamiento, del patógeno y de las condiciones del ambiente (Hernández et al., 2014). La multiplicidad de estos en un aislamiento, es una característica importante para su selección como agente de control biológico (Martínez et al., 2013).

La Figura 3 muestra que el tratamiento T7 (P.C + Safer soil ${ }^{\circledR}$ W.P) presentó diferencias significativas respecto a los demás tratamientos y el control regional (T10); mientras que el T6 no mostró diferencias frente al T4 y T8, pero si con los demás tratamientos y el T10. 


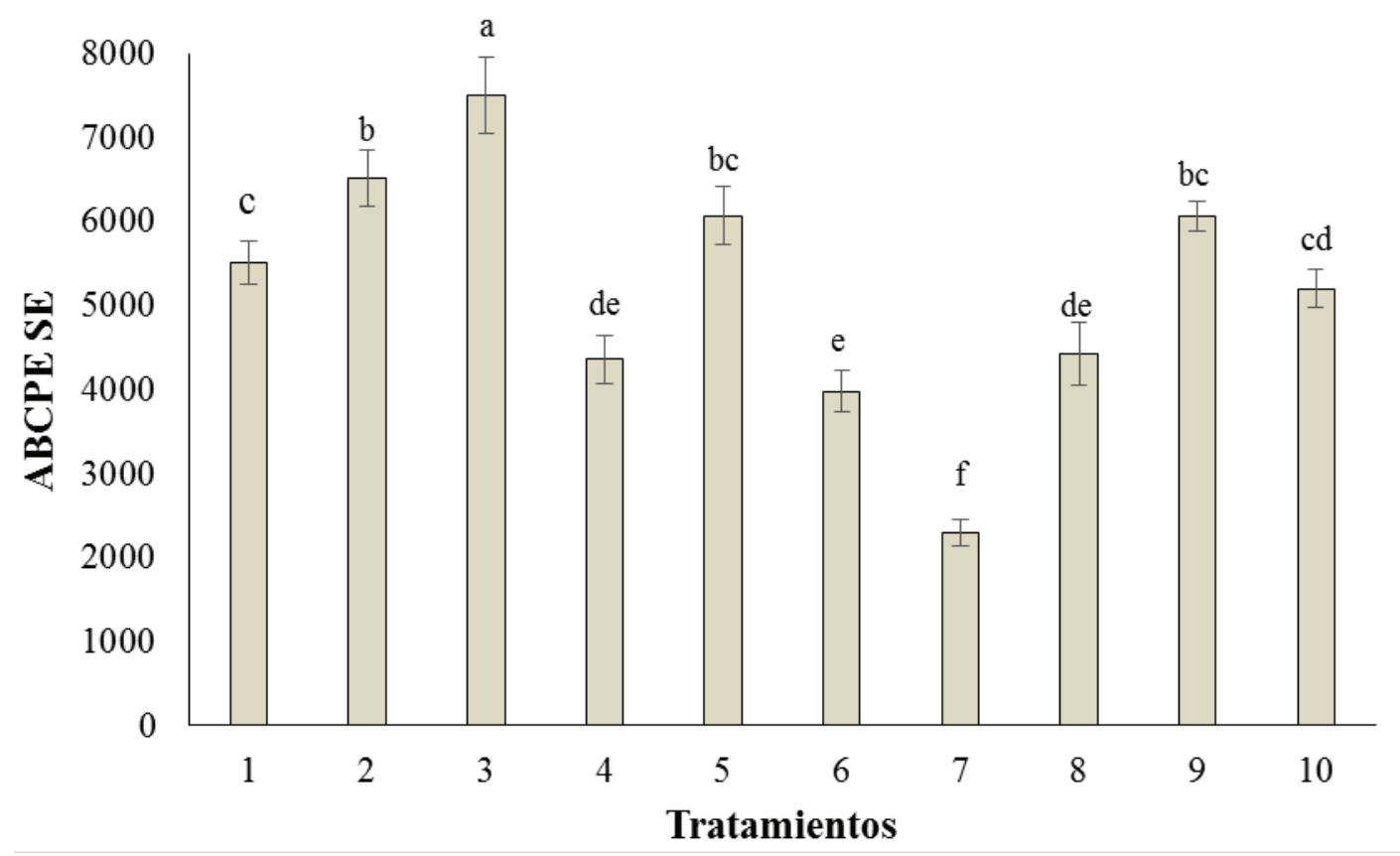

Figura 3. Área bajo la curva del progreso de la enfermedad (ABCPE) con base a severidad externa de $M$. roreri en respuesta a los tratamientos evaluados. Letras distintas indican diferencias significativas (Duncan; $\mathrm{P} \leq 0,01$ ). Los intervalos sobre cada barra representan el error estándar.

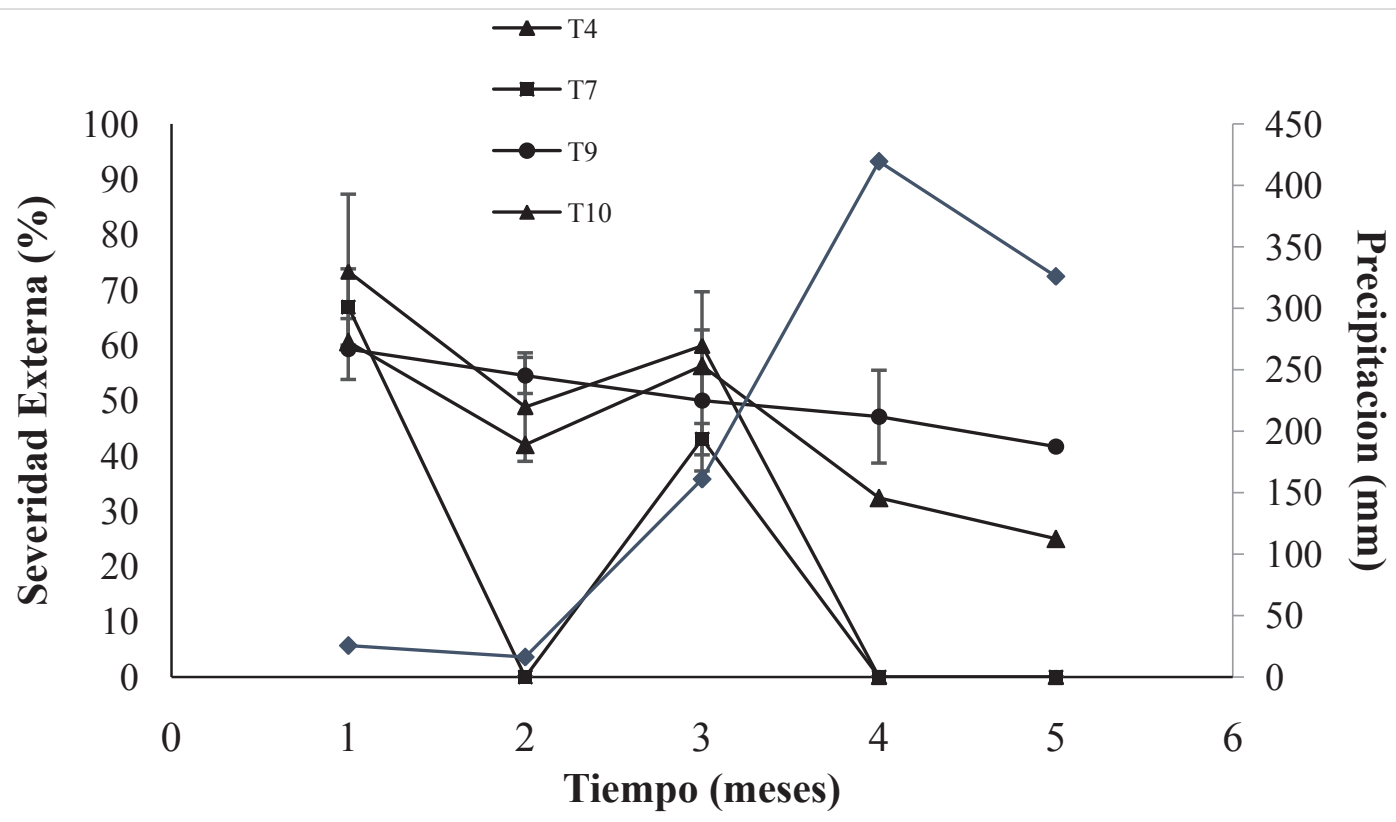

Figura 4. Relación entre la severidad externa de moniliasis sobre frutos de cacao y la precipitación en el tiempo, en presencia de los tratamientos T4, T7, control químico y testigo regional en condiciones de campo. Las barras sobre la curva representan el error estándar. 
La tendencia de la severidad externa de moniliasis a través del tiempo por los valores expresados, confirmó que el mayor control durante las veinticinco semanas del experimento se obtuvo con el T7 seguido por el T4, superando al T9 y T10 (Figura 4). Los tratamientos con el menor efecto antagónico fueron T2 y T3, seguidos de T9, T5, T1, T10, T8 y T6 (Figura 3).

Estos resultados concuerdan con Sánchez (2005), quien indicó el efecto antagónico in situ de cepas de Trichoderma viride y T. harzianum, las cuales redujeron la enfermedad causada por M. roreri en 21,5 y 19,3\%, respectivamente, con relación al testigo. Resultados similares obtuvieron Villamil (2011) y Villamil et al. (2015), quienes reportaron la disminución en la severidad externa de la enfermedad, con valores de 19,5 y 28\%, al evaluar aislamientos autóctonos de Trichoderma spp. H5 y H20, en su orden. De acuerdo con Torres de la Cruz (2010), un antagonista que inhiba el crecimiento micelial de $M$. roreri reduce su capacidad para infectar los frutos y causar la enfermedad. Trichoderma spp. presenta alta actividad competitiva frente a M. roreri, lo que tiene importancia especialmente en la fase saprofítica, ya que impide su desarrollo en el mismo sustrato al producir enzimas como viridina, trichodermina, exo y endogluconasas, celobiasas, quitinazas y el compuesto 6-pentyl- $\alpha$-pyrone (6PAP), que tienen actividad antifúngica (Hernández et al., 2014). Igualmente, se ha evidenciado el alto potencial antagónico de Trichoderma spp. como micoparásito al penetrar y causar lisis del micelio de Sclerotium cepivorum, Rhizotocnia solani, Sclerotium rolfsii, Phytophthora spp., Rosellinia spp., Fusarium oxysporum, Botrytis cinerea, Verticillium dahliae y Cladosporium fulvum en diferentes cultivos (Quiroz y Ferrera, 2008; Reyes et al., 2008; Torres et al., 2008).

Rendimiento de grano. No se presentaron diferencias estadísticas entre tratamientos para esta variable. Al comparar la producción de cacao en grano seco $\left(\mathrm{Kg}_{\mathrm{h}} \mathrm{ha}^{-1}\right)$, por efecto de la aplicación de hongos biocontroladores y el control químico en combinación con prácticas culturales en relación al control regional (T10:47,5 Kg.ha ${ }^{-1}$ ), durante 25 semanas de evaluación, se encontró que el mayor rendimiento lo presentó el T4:105,6 Kg.ha-1 seguido del T7 con 88,1 Kg.ha-1 ${ }^{-}$Las siguientes producciones obtenidas en su orden fueron: T3:87,5, T5:77,5, T2:69,4, T8:68,7, T1:65,6 Kg.ha-1 y T6:58,7 Kg.ha-1 ${ }^{-1}$ El menor rendimiento correspondió al control químico (T9) con 53,1 Kg.ha-1 ${ }^{-1}$ El bajo rendimiento del clon TCS01 obtenido en todos los tratamientos, se debe a que es un genotipo de tardío rendimiento el cual inicia su producción a partir de los 20 meses del establecimiento en campo, dependiendo principalmente de factores edafoclimáticos y el óptimo manejo agronómico así como fitosanitario del cultivo (Palencia y Agudelo, 2014). De acuerdo con Pinzón et al. (2007), estos materiales no superan los $100 \mathrm{~kg}^{-h^{-1}}$ de grano en peso seco, para el primer año de producción. Los mayores rendimientos mostrados por el T4 seguido del T7, representaron aumento en producción en relación al control regional de 50,1\% y 41,8\% respectivamente, lo cual coincide con Solis y Suárez (2005), quienes evidenciaron aumento en la producción hasta del 20\% frente al testigo productor utilizando el aislamiento autóctono Trichoderma koningiopsis en combinación con prácticas culturales.

Finalmente, se puede considerar que los microorganismos antagónicos que han surgido en coevolución con el patógeno presentan modificaciones específicas hacia éste, mejorando su adaptación biológica (Phillips y Wilkinson, 2007). El T4 (P.C + mezcla Trichoderma H3, H5, H20), se puede considerar como una alternativa promisoria dentro del manejo integrado de $M$. roreri, tomando importancia el componente microbiológico con los aislamientos nativos. El T7 (P.C + Safer soil ${ }^{\circledR}$ W.P) constituye la segunda opción para el productor, al presentar el mejor efecto biológico sobre la disminución en la incidencia y severidad externa de la 
enfermedad. Por su naturaleza, el control biológico no elimina, sino que disminuye las poblaciones del patógeno y como consecuencia, reduce la incidencia de la enfermedad, por lo tanto, debe emplearse en propuestas de manejo integrado con énfasis en el control cultural mejorado (Krauss et al., 2010).

Villamil et al. (2015) sugieren que para un mejor aprovechamiento del potencial del control biológico de M. roreri en cacao, la aplicación de los antagonistas microbianos debe integrarse con prácticas que prevengan la diseminación de la enfermedad como el uso de cortinas rompe vientos, desinfección de herramientas, evitar el traslado de material vegetal contaminado y el transporte de animales o el mismo hombre entre lotes de cacao. Igualmente, las medidas de control para el manejo integrado de plagas y enfermedades en el cultivo de cacao involucran la siembra de materiales genéticos de alta productividad con tolerancia o resistencia parcial a la enfermedad (Jaimes et al., 2011), podas de mantenimiento semestral, regulación del sombrío permanente, manejo trimestral de arvenses, nutrición balanceada del cultivo con base en análisis de suelos y foliar, sistemas adecuados de drenaje y remoción semanal de frutos enfermos (Rodríguez et al., 2005; Jaimes y Aránzazu, 2010). También se debe incluir la aplicación de mezclas de fungicidas protectantes (a base de cobre) y sistémicos (i.a. Azoxistrobin) en cultivos altamente productivos (Argüello, 2000; Ayala, 2008; Isaí, 2012).

\section{CONCLUSIONES}

La cepa Safer Soil ${ }^{\circledR}$ WP y la mezcla de Trichoderma spp. H3, H5 y H20 en combinación con prácticas culturales, presentaron el menor porcentaje de incidencia, severidad externa y área bajo la curva del progreso de la moniliasis, para las condiciones evaluadas. Estos tratamientos mostraron los mayores rendimientos de cosecha, sin diferencias significativas respecto al control químico y regional, representando una alternativa promisoria para la producción de cacao en la subregión occidental de Boyacá.

\section{AGRADECIMIENTOS}

Los autores expresan sus agradecimientos a COLCIENCIAS, programa Semilleros y Jóvenes Investigadores, doctorados nacionales y en el exterior de la convocatoria 617 de 2013; la Dirección de Investigaciones de la Universidad Pedagógica y Tecnológica de Colombia-UPTC, por la financiación de este proyecto; al profesor Jorge Orlando Blanco Valvuena por su orientación en la metodología y a todo el equipo de trabajo del Laboratorio de Control Biológico del GMBC.

\section{REFERENCIAS BIBLIOGRÁFICAS}

AYALA, M. 2008. Manejo integrado de moniliasis (Moniliophthora roreri) en cacao (Theobroma cacao L.) mediante uso de fungicidas, combinado con labores culturales. Tesis de grado ingeniero Agropecuario, Facultad Ingeniería en Mecánica y Ciencias de la Producción, Universidad de Guayaquil. 115p.

ARGÜELLO, 0. 2000. Tecnología para el mejoramiento del sistema de producción de cacao. CORPOICA. E.E. La Suiza. Impresores Colombianos, Bucaramanga. 144 p

BATEMAN, R., HIDALGO, E., GARCÍA, J., ARROYO, G., HOOPER, G., ADONIJAH, V. y KRAUSS, U. 2005. Aplication of chemical and biological agents for the management of frosty pod rot (Moniliophthora roreri) in Costa Rica cacao (Theobroma cacao L.). Annais of Applied Biology. 147:129 - 138.

BAILEY, B., BAE, H., STREM, M., CROZIER, J., THOMAS, S., SAMUELS, G., VINYARD, B. y HOLMES, K. 2008. Antibiosis, mycoparasitism, and colonization success for endophytic Trichoderma isolates with biological control potential in Theobroma cacao L. Biological Control. 46(1):24 - 35. 
Centro de Investigaciones del Café (CENICAFÉ). 1994. Práctica Producción artesanal de Hongos Entomopatógenos. Chinchiná, Noviembre: 19-22 (mimeografiado) 2 p.

CÁRDENAS, C. y GIRALDO, J. 1986. Evaluación de la respuesta de algunos cultivares del cacao (Theobroma cacao L.) a Moniliophthora roreri mediante dos métodos de inoculación en frutos y en semilla en estado radicular. Trabajo de grado Ingeniero Agrónomo, Facultad de Agronomía, Universidad de Caldas. Manizales. 107 p.

FEDERACIÓN NACIONAL DE CACAOTEROS (FEDECACAO). 2015. Estadísticas de la producción de cacao en grano en Colombia. En: http://www.fedecacao.com.co; consulta: marzo 2015.

GÓMEZ, K. y GÓMEZ, A. 1984. Statistical procedures for agricultural research. Second edition, John Wiley and Sons, New York. 680 p.

GUERRERO, Y., HERNÁNDEZ, A., HEYDRICH, M., EL JAZIRI, M. y HERNÁNDEZ, A. 2012. Management of black pod rot in cacao (Theobroma cacao L.). Fruits. 67:41 - 48.

HERNÁNDEZ, A., RUÍZ, Y., ACEBO, Y., MIGUÉLEZ, Y. y HEYDRICH, M. 2014. Antagonistas microbianos para el manejo de la pudrición negra del fruto en Theobroma cacao L. Estado actual y perspectivas de uso en Cuba. Protección Vegetal. 29(1):11 - 19.

HEBBAR, P. 2007. Cacao Diseases: A global perspective from an industry point of view. Phytopath. 97:1658 1663.

ISAÍ, D. 2012. Evaluación de fungicidas sistémicos y de contacto en el control de la Moniliasis (Moniliophthora roreri) del Cacao (Theobroma cacao L.) Tabasco. Tesis de grado Magister Ciencias, Posgrado Producción Agroalimentaria en el Trópico, Colegio de Postgraduados. Tabasco. $86 \mathrm{p}$.

JAIMES, Y. y ARANZAZU, F. 2010. Manejo de las enfermedades del cacao (Theobroma cacao L.) en Colombia, con énfasis en monilia (Moniliophthora roreri). Primera edición. Corpoica C.I. La Suiza, Bogotá D.C. 90 p.
JAIMES, Y., ARÁNZAZU, F., RODRÍGUEZ, E. y MARTÍNEZ, N. 2011. Behavior of introduced regional clones of Theobroma cacao L. toward the infection Moniliophthora roreri in three different regions of Colombia. Agronomía Colombiana. 29(1):171 - 178.

KRAUSS, U., HIDALGO, E., BATEMAN, R., ADONIJAH, V., ARROYO, C., GARCÍA, J., CROZIER, J., NEIL, A., MARTIJN TEN HOOPEN, G. y HOLMES, K. 2010. Improving the formulation and timing of application of endophytic biocontrol and chemical agents against frosty pod rot (Moniliophthora roreri) in cocoa (Theobroma cacao). Biological Control. 54(3):230 - 240.

LUCERO, A., PEÑA, L. y BACCA, T. 2004. Evaluación de la actividad biocontroladora de Beauveria bassiana y Metarhizium anisopliae sobre larvas de Ancognatha scarabaeiodes (Coleóptera: Scarabaeidae). Ciencia \& Tecnología Agropecuaria. 5(1):43 - 48.

MARTÍNEZ, B., INFANTE, D. y REYES, Y. 2013. Trichoderma spp. y su función en el control de plagas en los cultivos. Protección Vegetal. 28(1):1 - 11.

MARTÍNEZ, B., REYES, Y., INFANTE, D., GONZÁLEZ, E., BAÑOS, H. y CRUZ, A. 2008. Selección de aislamientos de Trichoderma spp. candidatos a biofungicidas para el control de Rhizoctonia sp. en arroz. Protección Vegetal. 23(2):118 - 125.

OSORIO, R. 2010. Estudio del efecto de Trichoderma harzianum en el control de Moniliophthora roreri en plantas de Theobroma cacao en la provincia de Esmeraldas. Tesis de Grado Ingeniero Agroindustrial, Facultad Ingeniería Química y Agroindustrial, Escuela Politécnica Nacional. Quito. 125 p.

PROEXPORT, 2012. Cacao colombiano fino y de aroma. Bogotá D.C. 15 p.

PINZÓN, J., ROJAS, J. y ROJAS, F. 2007. Guía técnica para el cultivo de Cacao. Segunda edición, Ediciones LCB Ltda, Bogotá D.C. Colombia. 192 p.

PALENCIA, C. y AGUDELO, G. 2014. Protocolo de siembra de clones de cacao en sistemas agroforestales y jardines clónales para Boyacá y Santander. Manual técnico, Edición CORPOICA-C.I. La Suiza, Bucaramanga. $7 \mathrm{p}$. 
PÉREZ, W. y FORBES, G. 2008. El Tizón Tardío de la papa. Manual técnico, Edición Comercial Grafica Sucre, Centro Internacional de la papa (CIP), Lima. 12 p.

PHILLIPS, W. y WILKINSON, M. 2007. Frosty pod of Cacao: A disease with limited geographic range but limited potential for damage. Phytopathology. 97:1644 - 1647.

PHILLIPS, W., AIMES, M. y WILKINSON, M. 2007. Biodiversity and biogeography of the cacao (Theobroma cacao) pathogen Moniliophthora roreri in tropical America. Plant Pathology. 56(6): 911 - 922.

QUIROZ, V. y FERRERA, C. 2008. Antagonismo in vitro de cepas de Aspergillus y Trichoderma hacia hongos filamentosos que afectan al cultivo del ajo. Revista Mexicana de Micología. 26:7 - 34.

REYES, Y., MARTÍNEZ, B. y INFANTE, D. 2008. Evaluación de la actividad antagónica de trece aislamientos de Trichoderma spp. sobre Rhizoctonia sp. Protección Vegetal. 23(2):112 - 117.

RODRÍGUEZ, E., MUJICA, J. y CUBILLOS, G. 2005. Manejo integrado de la moniliasis en el cultivo del cacao. Edición CORPOICA, Litografía Andrés Bello, Bucaramanga. $25 \mathrm{p}$.

RODRÍGUEZ, J., VELANDIA, J. y VITERI, S. 2010. Evaluación de microorganismos aislados de gallinaza por su potencial para el biocontrol de Fusarium (F. oxysporum) en plántulas de Uchuva (Physalis peruviana). Revista de la Facultad Nacional de Agronomía de Medellín. 63(2):5499 - 5509.

SÁNCHEZ, M. 2005. Efecto antagónico de Trichoderma harzianum y Trichoderma viride sobre Moniliophthora roreri Cif \& Par en cacao. Tesis de Grado Agronomía, Facultad de Ciencias Agropecuarias, Universidad Nacional de Ucayali. 55 p.

SHANER, G. y FINNEY, R. 1977. The effect of nitrogen fertilization on the expression of slow-mildewing resistance in Knox wheat. Phytopathology. 67,1051 - 1056. Suárez, L. y Rangel, A. 2013. Aislamiento de microorganismos para el control biológico de Moniliophthora roreri. Acta Agronómica. 62(4):370 - 378.
SOLIS, K. y SUÁREZ, C. 2005. Uso de Trichoderma spp. para control del complejo Moniliasis-Escoba de Bruja del cacao en Ecuador. Instituto Nacional Autónomo de Investigaciones Agropecuarias, Boletín técnico, Quevedo. $8 \mathrm{p}$.

TORRES, E., IANNACONE, J. y GÓMEZ, H. 2008. Biocontrol del moho foliar del tomate Cladosporium fulvum empleando cuatro hongos antagonistas. Bragantia. 67(1):169 - 178.

TORRES DE LA CRUZ, M. 2010. Progreso temporal y manejo integrado de la moniliasis Moniliophthora roreri Cif y Par. (Evans et al.) del cacao (Theobroma cacao L.) en Tabasco. Tesis doctoral en Fitosanidad, Facultad posgrado de Fitosanidad, Universidad Juárez Autónoma de Tabasco. 99 p.

VILLAMIL, J. 2011. Evaluación de hongos y bacterias en la capacidad antagónica a monilia (Moniliophthora roreri) en cacao (Theobroma cacao L.) in vitro y en campo. Trabajo de grado ingeniero Agrónomo, Facultad de Ciencias Agropecuarias, Universidad Pedagógica y Tecnológica de Colombia. Tunja.78 p.

VILLAMIL, J., BLANCO, J. y VITERI, S. 2012. Evaluación in vitro de Microorganismos Nativos por su Antagonismo contra Moniliophthora roreri Cif \& Par en Cacao (Theobroma cacao L.). Revista de la Facultad Nacional de Agronomía de Medellín. 65(1):6305 - 6315.

VILLAMIL, J., VITERI, S. y VILLEGAS, W. 2015. Aplicación de Antagonistas Microbianos para el Control Biológico de Moniliophthora roreri Cif \& Par en Theobroma cacao L. bajo condiciones de campo. Revista de la Facultad Nacional de Agronomía de Medellín. 68(1):7441 - 7450.

VÉLEZ, P., POSADA, F., MARÍN, P., GONZÁLEZ, M., OSORIO, E. y BUSTILLO, A. 1997. Técnicas para el control de calidad de formulaciones de hongos entomopatógenos. Boletín Técnico, No. 17. Cenicafé, Chinchiná, 47 p. 